\title{
EL MALESTAR EN LA RELACIÓN MÉDICO- PACIENTE
}

\author{
Gabriela Manitta
}

\begin{abstract}
Resumen: Lo que se plantea en torno al malestar en la relación médico- paciente es la posibilidad de reconocer la locura que engendran algunas normas, tales como aquella que pretende transformar la acción médica en una operación de autómata.

El médico puede asumir una posición diferente si está dispuesto a preservar en sí el lugar de su propia ignorancia acerca de la particularidad inalienable de cada enfermo, de esa historia individual en la que anida el síntoma.

Pues aun cuando se esté en condiciones de afirmar que se han respetado los derechos del paciente, nada se sabe de él. Es necesario que el médico pueda situarse frente al sujeto como frente a un misterio para poder reconocer la particularidad de éste y para que el paciente pueda reconocerse en su particularidad.
\end{abstract}

Palabras clave: relación médico- paciente, otro, particularidad, síntoma, pregunta, ignorancia, tecnociencia, palabra, norma

\section{UNEASINESS IN THE PHYSICIAN-PATIENT RELATIONSHIP}

\begin{abstract}
The possibility to recognize the madness that certain norms generate, such as the one that pretends to transform the medical action into an automaton's operation is stated around the uneasiness in the physician-patient relationship.

Physicians may assume a different position if they are willing to keep in themselves the sight of their own ignorance about the inalienable particularity of each sick person, and of the individual history that shelters the symptoms.

Because even though we can assert that the patient's rights have been respected, we know nothing about him. In order to recognise his patient's particularity, and for the patient to acknowledge himself in that particularity, the doctor has to face him as if he was facing a mystery.
\end{abstract}

Key words: physician-patient relationship, another one, particularity, symptom, question, ignorance, technoscience, word, norm

\section{O MAL ESTAR NA RELAÇÃO MÉDICO-PACIENTE}

Resumo: O que se coloca em torno do mal estar na relação médico-pacinte é a possibilidde de reconhecer a loucura provocada por algumas normas, tais como aquela que pretende transformar a ação médico numa operação autômata. $\mathrm{O}$ médico pode assumir uma posição diferente se está disposto a preservar em si, o lugar de sua própria ignorância em relação à singularidade inalienável de cada doente, de sua história individual que se localiza no sintoma.

Mesmo quando se está em condições de afirmar que os direitos do paciente foram respeitados, nada se sabe dele. É necessário que o médico se situe frente ao sujeito, estando frente a um mistério para poder reconhecer a singularidade deste, e para que o paciente também possa reconhecer-se em sua singularidade.

Palavras chaves: relação médico-paciente, outro, singularidade, sintoma, pergunta, ignorância, tecnociência, palavra, norma

\footnotetext{
* Licenciada en Psicología. Alumna de la Maestría en Bioética en la Universidad Nacional de Cuyo. Argentina
} Correspondencia: gmanitta@hotmail.com 


\section{Introducción}

En este trabajo pretendo interrogar la relación médico-paciente a partir del malestar que en su configuración actual se evidencia. No busco alcanzar respuestas abarcadoras y tranquilizantes, pues considero que sólo en la incomodidad de la pregunta es posible el movimiento de búsqueda.

Comenzaré recorriendo algunos conceptos sobre la alteridad y su misterio, y sobre la constitución del sujeto. Luego puntualizaré cuestiones referidas específicamente al vínculo entre el médico y el paciente. Finalmente, intentaré ubicar una posición diferente para ambos frente a la tecnociencia y la norma.

\section{Del sufrimiento al sujeto}

Emmanuel Levinas afirma que en el dolor físico se encuentra inequívocamente el compromiso con la existencia. "En el sufrimiento se produce la ausencia de todo refugio. Es el hecho de estar directamente expuesto al ser. Procede de la imposibilidad de huir y de retroceder. Todo el rigor del sufrimiento consiste en esa imposibilidad de distanciamiento. Supone el hecho de estar acorralado por la vida y por el ser. En este sentido, el sufrimiento es la imposibilidad de la nada(1)".

Además de la apelación a una nada imposible, en el sufrimiento está presente también la proximidad de la muerte, cuya incógnita se presenta como el correlato de la experiencia de la imposibilidad de la nada. Levinas señala que la propia relación con la muerte no puede tener lugar bajo la luz; el sujeto entra en una relación con algo que no proviene de él. Se trata de la relación con el misterio.

La muerte anuncia un acontecimiento del que el sujeto no puede adueñarse, pues es algo que escapa a su comprensión. Entonces con la muerte no es posible una relación de objeto, por lo que respecto de ella el sujeto deja de serlo: "...de ella [de la muerte] es de lo que precisamente no sabemos nada(1, p. 113)".

Por ser incomprensible, la muerte escapa a todo presente. El "ahora", para Levinas, supone que el sujeto es el dueño de lo posible, de captar lo posible. La muerte nunca es ahora. Es una relación única con el futuro. Por lo tanto, cuando la muerte existe el sujeto ya no está, pues no se encuentra en disposición de captar nada. Y es en ese sufrimiento (donde se capta la vecindad de la muerte) que se produce el paso del sujeto de la actividad a la pasividad.

Levinas aclara que este paso no se produce en el instante en el que aún podemos captar el sufrimiento, en el que aún somos sujetos del sufrimiento, sino en el llanto y el sollozo en los que éste desemboca. "Morir es retornar a ese estado de irresponsabilidad, morir es convertirse en la conmoción infantil del sollozo(1, p. 113)".

\section{La constitución del sujeto}

En el inicio de la vida no hay Yo. Lacan designa como "estadio del espejo" a la instancia necesaria para la formación del Yo en el sujeto humano. Para que éste se constituya será necesaria la asunción de una imagen por mediación del Otro.

El Otro de los primeros cuidados le otorga al bebé humano una imagen de sí que lo rescata de la discordancia primordial en la que se encuentra, debido a incoordinación motriz propia de la prematuración del nacimiento.

El sujeto, entonces, se precipita de la insuficiencia original a la anticipación. Por medio de la identificación con lo que Lacan designa como "Yo ideal" el sujeto humano se desliza de una imagen fragmentada del propio cuerpo a una forma ortopédica de su totalidad. 
Esta forma que le ofrece el Otro, y con la que el sujeto se identifica, es siempre enajenante, pero fundamental para la supervivencia; porque se trata de la matriz simbólica en la que el Yo se precipita.

A lo largo de la vida hay situaciones que nos remiten nuevamente a esa situación de indefensión y fragmentación primordial. La enfermedad y el padecimiento físico son algunas de ellas, por esto van siempre acompañadas de un monto de angustia que varía de acuerdo con las características singulares.

La enfermedad nos hace presente la imposibilidad de garantías, la finitud y la fragilidad de la vida; fragmenta el cuerpo, lo hace ajeno, desconocido. Nos pone ante la muerte y volvemos a experimentar algo que ya conocimos en el inicio de nuestra vida: la indefensión.

El sujeto se dirige entonces al médico como a ese Otro que puede devolverle una imagen de sí completa, sin grietas por donde asome la angustia. En el diagnóstico el médico otorga una posibilidad de identificación, que para cada sujeto se juega de forma singular. Por ello, para alguien "ser infértil", por ejemplo, es ya una forma de existencia, es un significante con el que es posible identificarse y que rescata al sujeto de la nada, del "no ser".

Para el sujeto humano lo posible siempre estará del lado de lo simbólico, pues se trata de un sujeto de palabra que, como tal, sólo encuentra posibilidades en las significaciones. La muerte no puede significarse, queda fuera del registro simbólico porque no hay un significante que la presentifique para el sujeto que habla.

La posibilidad de la muerte provoca angustia justamente porque no puede asumirse, no se la puede aprehender en el presente, es pura ausencia.

\section{El otro semejante}

Para abordar esta cuestión, podemos preguntarnos ¿qué es lo Otro? Y también ¿quién es el otro? Preguntas diferentes, por cierto.

Para Levinas, la proximidad de la muerte indica que estamos en relación con algo que es absolutamente otro. Lo Otro que así se anuncia no existe del mismo modo que el sujeto. $\mathrm{Su}$ poder sobre el existir del sujeto es misterioso, no ya desconocido sino incognoscible.

La muerte es lo absolutamente Otro de lo cual el sujeto no puede saber. Como alteridad radical nos acerca al misterio de la relación con los otros, en el sentido de que cada semejante es también un misterio, una "otredad" opaca.

"La relación con otro no es una relación idílica y armoniosa de comunión ni una empatía mediante la cual podamos ponernos en su lugar: le reconocemos como semejante a nosotros y al mismo tiempo exterior; la relación con otro es una relación con un Misterio (1, p. 116)."

En esa relación con los demás al sujeto le acontece algo, que no asume pero con lo que se enfrenta en cierto modo. En el cara a cara con los otros se produce un encuentro con un rostro en el que el otro se da y al mismo tiempo se oculta.

Entonces, la relación con otro comporta algo más que relaciones con el misterio, ya que se aborda al otro en la vida corriente. "Uno es para el otro lo que el otro es para uno; no hay lugar excepcional para el sujeto. Se conoce al otro por empatía, como a un otro-yo mismo(1, p.126)".

Sin embargo, en el interior de la relación con otro que caracteriza la vida social aparece la relación como no recíproca, es decir, como relación que quebranta la contemporaneidad. "El otro en cuanto otro no es solamente un alter ego: es algo que yo no $\operatorname{soy}(1, p .127)$ ". 
Podríamos decir que lo cotidiano vela el misterio, lo esconde detrás de una pantalla. Desde aquí es posible comprender lo que en una primera lectura parecería una contradicción. Pues, por un lado, Levinas nos dice que la relación con otro no es una en la cual podamos conocerlo por empatía y, al mismo tiempo, afirma que se conoce al otro como a otro-yo mismo.

Lo Otro que el otro semejante nos representa es siempre misterio, presencia inasible, incognoscible. De lo Otro podemos tener alguna pista, alguna señal en la angustia y el sollozo. Pero no hay relación posible ya que en su presencia el sujeto pierde su estatus de tal.

Es decir, para que sea posible el encuentro con otro será necesario reconocerle su alteridad irreductible a la semejanza con el yomismo. "A partir de Platón el ideal de lo social se buscará en un ideal de fusión. Se pensará que, en su relación con otro, el sujeto tiende a identificarse con él, abismándose en una representación colectiva, en un ideal común. Es la comunidad que dice 'nosotros', la que, vuelta hacia el sol inteligible, hacia la verdad, siente al otro junto a sí y no frente a sí. Una colectividad necesariamente establecida en torno a un tercer término que sirve de intermediario(1, p. 137)".

Levinas opone a esta colectividad del "junto a", la colectividad "yo-tú". No se trata de una participación en un tercer término -ya sea una persona, una verdad, una obra o una profesión. Se trata de una colectividad que no es una comunión. Es el cara a cara sin intermediarios donde la proximidad del otro no anula la distancia.

El "junto a" implica desconocer la distancia, tomar al otro semejante en calidad de objeto y creer que podemos conocerlo como un reflejo del sí-mismo en el espejo. Implica también olvidar que el yo es lugar de desconocimiento, dado que el sujeto nunca puede dar cuenta acabada de sí mismo, aún cuando le sea posible producir un efecto de verdad acerca de sí.

En el "junto a" es donde tienen lugar la rivalidad y la empatía, por la identificación con la imago del semejante. Pues lo idéntico no deja lugar a la diferencia y si el otro es igual al sí mismo no se lo reconoce en su alteridad. "El otro no es un ser con quien nos enfrentamos, que nos amenaza o que quiere dominarnos. El hecho de que sea refractario a nuestro poder no representa un poder superior al nuestro. Todo su poder consiste en su alteridad. Su misterio constituye su alteridad(1, p.130)".

Levinas explica que no consideramos al otro inicialmente como libertad, ya que en este caso la comunicación fracasa de antemano. Porque la única relación posible con una libertad es la sumisión o el avasallamiento. Y en ambos casos una de las dos libertades queda aniquilada. Lo que caracteriza inicialmente al otro no es la libertad. La esencia del otro es la alteridad. Del mismo modo que con la muerte, no nos enfrentamos en este caso con un existente, sino con el acontecimiento de la alteridad.

Por esto, insiste, la alteridad no es pura y simplemente la existencia de otra libertad junto a la mía. El otro permanece de alguna manera ausente en su misterio y, precisamente por ello, se hace presente como otro. No es posible conocerlo, poseerlo o aprenderlo pues entonces ya no sería otro.

En el cara a cara que implica el "yo-tu" se hace posible un espacio propiamente humano donde cada sujeto preserva el uso de la palabra para sí y para el otro. La palabra le otorga a cada uno su lugar. En este sentido el diálogo es, en sí mismo, exigencia de alteridad y, por ello, implica siempre a otro constituyendo una renuncia a la agresividad entendida como anulación de las diferencias. 
El diálogo exige reconocer al otro como sujeto diferente al yo, accesible en su semejanza pero, a la vez, lejano en su propio misterio. "Sólo un sujeto puede comprender un sentido; inversamente, todo fenómeno de sentido implica un sujeto(2)".

El sentido se construye entre sujetos, ya que es el otro quien sanciona la significación de lo dicho. Por ello, el mensaje no es algo que se produzca de una vez y para siempre, sino que va cobrando nuevas significaciones en tanto circula entre los sujetos.

\section{Entre el médico y el paciente}

Podría decirse que, hasta hace algunos años, entre el médico y el paciente estaban la enfermedad y el síntoma. En la actualidad, entre ellos hay, además, una pregunta por la ética. Siguiendo a Castoriadis, "en el peor de los casos la palabra ética se utiliza como eslogan y en el mejor, sólo es signo de un malestar y una pre$\operatorname{gunta}(3)$ ".

El síntoma es un malestar que se manifiesta como una pregunta que el sujeto no acierta a responder, como una opacidad, un vacío de palabra, una incógnita que molesta porque aparece como algo extraño, ajeno. La ética también denuncia un malestar particular de nuestra época. Podemos situar este malestar en el "entre-sujetos" y decir, con palabras de Engelhardt, que "existe una tensión fundamental entre el objetivo de lograr el bien de las personas y el respeto hacia ellas como agentes morales libres y responsables. Existen desacuerdos fundamentales acerca de quién debe definir el bien de las personas, cómo y con respecto a qué norma(4)."

Del mismo modo, al observar vínculos particulares nos damos cuenta de que se pueden verificar malestares tanto en las relaciones de pareja como en las de padre-hijo, madre-hijo, maestro-alumno, jefe-empleado, etc. Se trata de un "estar-mal", o mejor dicho, un "estar-malcon otro" generalizado.

La declinación del padre y de los ideales ha afectado la distribución de lugares y, con ello, emergen grandes dificultades para identificarse a un "tipo ideal", a una manera de ser hombre, mujer, padre, madre, discípulo, hijo, etc.

Entre las relaciones que albergan un malestar, la relación médico-paciente ha sido una de las más estruendosas, con acusaciones, denuncias, reclamos, sentencias, códigos de ética, compromisos y tratados mundiales de por medio. En principio, lo que hay en esta relación son dos sujetos, uno de ellos a quien se le supone un saber (el médico) y otro que, en razón de ese supuesto, se dirige al primero con una demanda (el enfermo).

Pero si antes el escenario de este encuentro era, fundamentalmente, la enfermedad, hoy las cosas han cambiado. Se han modificado las demandas porque se ampliaron las ofertas; o bien, las que se ampliaron fueron las demandas porque se modificaron las ofertas. "En consecuencia, se amplía el espectro de la atención de la salud -que incluye desde el consejo genético a la cirugía cosmética- y los fines de la medicina -tradicionalmente reparadora y cada vez más modeladora de la naturaleza humana- se someten al análisis de beneficios(5)".

En la época de la medicina "cosmética" el alcance de la intervención médica no sólo "debe" orientarse a reparar, sino, sobre todo, a devolver la felicidad. El sujeto exige que se le otorgue el beneficio de acceder a un estado libre de molestias y de deseo peligrosamente saciado. Donde el paciente decía "el doctor hizo todo lo que pudo" actualmente hay demanda por mala praxis, pues hoy "todo es posible"; es cuestión de encontrar al médico indicado con 
los aparatos adecuados. Entre el médico y el paciente hay en la actualidad una presencia opaca que se ha dado en llamar "tecnociencia".

J. Ladrière nos dice que el carácter más esencial de la tecnociencia es su capacidad para constituir un universo que se encuentra en interacción con el natural, pero que se construye como una totalidad sui generis, cada vez más autónomo, imponiendo de manera casi obligatoria sus propias leyes de funcionamiento a la acción humana. "En realidad, es la acción humana la que engendra la tecnociencia, pero, creándola, se convierte en deliberadamente dependiente de las posibilidades que ésta le ofrece, aunque también de las posibilidades que se le abren y de las limitaciones que ello implica(6)".

Es necesario, en la actualidad, establecer una suerte de mediación entre el médico, el paciente y la tecnociencia. Mediación en el sentido de hacer lugar a la palabra en un reino donde imperan el silencio y la asepsia, donde los datos son mediciones y éstas dejan fuera a lo que no responda a un criterio de eficiencia.

"¡Medida en todas las cosas! La expresión cambia de sentido. La ética de la medida es griega, especialmente aristotélica, y significaba la necesidad de estar en el justo medio entre dos polos extremos. De ahora en más significa que la única ética posible es medir para comparar entre los efectos esperados de una acción y los progresos efectivamente realizados [...] En este marco neo-utilitarista, el único bien auténticamente reconocido sería la mejor relación calidad-precio(7)."

Antes que la relación médico-paciente, lo que se modifica es la relación de ambos con la salud. Del lado del médico en cuanto debe administrar beneficios y responder a los criterios de eficacia importados del mercado; del lado del paciente vemos que hoy el poder de la ciencia brinda al sujeto la posibilidad de recurrir al médico a pedirle su cuota de beneficios con un objetivo preciso inmediato.

\section{La pregunta en el cuerpo}

El síntoma genera en el sujeto una pregunta, un punto de opacidad que no acierta a descifrar y con el cual se dirige al médico. "El médico es el depositario del saber sobre el cuerpo, en esa posición lo instituye el sujeto(8)".

Cuando hablamos del cuerpo hacemos referencia a algo que está más allá de las vicisitudes del organismo. En lo real tenemos un organismo que, eventualmente, presenta alteraciones -anatómicas o funcionales- que son objeto de medicina. Ahora bien, si de lo que hablamos es de un sujeto humano, entonces además de un organismo hay un cuerpo.

Cada paciente tiene una forma particular de enfermar y de sanar, más allá de que los diagnósticos y tratamientos sean generalizables. Se trata de lo que permanece fuera de las mediciones y las estadísticas, de la manera singular de manifestar el sufrimiento y padecerlo. Se trata del síntoma que sólo es posible en el sujeto que habla.

Sin embargo, el sujeto mismo desconoce que su cuerpo está cifrado en un discurso específico; ignora que su cuerpo se utiliza según un código inconsciente y que ese código no es el del cuerpo médico. "Antes de que aparezca y se asuma ese discurso particular, el paciente se presenta con su cuerpo, cuya marca es determinado síntoma $(8, p .162)$ ”.

Lo que el paciente pide, más allá de la cura, es un saber acerca de su cuerpo. En ese punto, y a través del síntoma, dice Michael Tort, la demanda del sujeto, como demanda de un saber, encuentra la respuesta médica que la captura en su saber. Muchas veces esa respuesta implica un contrato de somatización inducido 
por el deseo del médico y su exclusivo interés en el funcionamiento real de los órganos y funciones. Dicha somatización, siempre según Tort, está implícita en toda demanda u oferta de intervención en el cuerpo y corresponde al proceso por el cual la demanda del sujeto es tomada "al pie de la letra" para dar lugar a una intervención sobre el mismo cuerpo.

Es decir, el paciente pregunta con un síntoma y el médico responde desde el espacio clínico con una determinación anatómica/ funcional, producto de su saber sobre los cuerpos no sobre los sujetos. El diagnóstico abarca la extensión del dolor y sus manifestaciones, pero deja fuera la dimensión del sujeto que con y en ese cuerpo habla.

Al mismo tiempo, existe lo que Tort llama "somatización solicitada". Es el proceso por el cual queda "entendido" que lo que el paciente trajo -y expuso a examen, diagnóstico e intervención- es el cuerpo y no el sujeto de palabra que requiere ser escuchado.

La somatización solicitada es una especie de contrato en el cual el sujeto dirige su demanda al médico y recibe de éste la certeza de que su padecer será tomado al pie de la letra. El paciente exhibirá su cuerpo a condición de desconocer eso "otro" referente a su condición de sujeto deseante, sufriente, sujeto de palabra. El médico, entonces, cae en la trampa que implica la transacción de ofrecer y recibir el cuerpo sólo como un puro organismo que no funciona.

Este pacto desvía la cuestión de deseo de sujeto y aliena el padecer a una respuesta tecnocientífica. Ahora bien, como se dijo antes, el médico es depositario de un saber y se espera de él que responda de alguna manera con el fin de devolverle al sujeto el bienestar perdido o, en última instancia, atenuar el dolor.

Entonces, ¿dónde está el límite en que el médico debe actuar y a qué debe responder?
Lacan nos dice que es en el registro del modo de respuesta a la demanda del enfermo donde está la posibilidad de supervivencia de la posición propiamente médica. Desde el psicoanálisis se apunta que no existe una correspondencia unívoca entre la demanda y el deseo, que cuando cualquiera nos pide algo, esto no es para nada idéntico e, incluso, a veces es diametralmente opuesto a aquello que desea.

El deseo es siempre inconsciente, no articulable en palabras, desconocido para el sujeto mismo. Por ello, podemos decir que toda demanda es un pedido complejo, que muestra y esconde, señala y engaña. El enfermo recurre a veces al médico para que lo autentifique en su condición de enfermo, o para que se lo trate de tal modo que se le permita seguir siendo un enfermo bien instalado en su enfermedad, aún cuando lo que explícitamente pide es la cura.

Esto significa que la tarea del médico no implica exclusivamente la administración de lo que se encuentra al alcance de la mano -un aparato quirúrgico o un antibiótico-, sino que más allá, o más acá, de éstos objetos existe otra dimensión que es necesario sostener. Atender a la dimensión de la demanda en toda su complejidad sólo es posible si el médico está dispuesto a preservar en sí el lugar de la ignorancia, de su propia ignorancia acerca de la particularidad inalienable de cada enfermo, de esa historia individual en la que anida el síntoma. De ésta manera, el médico hace posible la emergencia de una pregunta del paciente acerca de su síntoma. "Entramos ahí en un espacio sorprendente, no avalado por el saber, el único donde el deseo puede sorprender(se)(8, p.161)".

Cada vez que un paciente consulta por su síntoma, el médico tiene en sus manos la posibilidad de plantear una pausa en la carrera. Debe responder, es cierto. Pero no está obligado a responder siempre con una oferta. Por esta vía se pueda quizás pesquisar también algo del en- 
gaño que implica la pantalla tecnocientífica. "Pues la ciencia no es incapaz de saber qué puede; pero ella, al igual que el sujeto que engendra, no puede saber qué quiere(9)".

La ciencia sabe de operaciones, y lo que se está produciendo en el seno de la relación médico-paciente es una sustitución de la acción médica por la operación tecnocientífica. Tomando las definiciones que propone J. Ladrière, podemos definir a la operación como un proceso que se ejecuta de forma mecánica, según reglas establecidas, que la separa completamente de la significación. La acción es un proceso que se ejecuta en el marco de una intención y que se guía de manera continua por lo que ésta sugiere. "El programa operatorio es aplicable de manera indefinida $\mathrm{y}$, en este sentido, es universalizable. La acción, al contrario, es siempre singular(6, p.209)”.

\section{Conclusiones}

Laurent señala que nos encontramos sumergidos en la búsqueda inquieta por justificar nuestra existencia, en una época en que el ideal se reduce a la eficacia: "Cada uno debe funcionar en el nivel más eficaz posible(7, p.152)". Actualmente, el nivel de funcionamiento que se impone al sujeto es una derivación del que se supone en la máquina: la operación indicada con el menor costo posible y el mayor beneficio esperable.

También J. Ladrière nos pone en aviso sobre este punto: "Por una especie de inversión, llega un momento en que el sistema de necesidad, determinado por las propiedades somáticas y psíquicas del ser humano, no domina ya el desarrollo tecnológico, sino que es el devenir de la misma tecnología el que rige el sistema de necesidades(10)".

Y si consideramos que el ideal de la tecnología puede compararse al de la ciencia -en el sentido de que siempre buscará saber qué puede aunque no sepa qué quiere- encontramos la posición del sujeto más que comprometida. Sólo al sujeto humano le está reservada la posibilidad del deseo, pero éste, que rescata la particularidad del sujeto, representa un peligro para el ideal de eficacia.

"El poder se ejerce ahora a través de maquinarias que organizan directamente los cerebros (en los sistemas de comunicación, las redes de información, etc.) y los cuerpos (en los sistemas de asistencia social, las actividades controladas, etc.) con el propósito de llevarlos hacia un estado autónomo de alienación, de enajenación en el sentido de la vida y del deseo de creatividad(11)". Cuerpos y cerebros quedan así deshabitados, pues donde el deseo singular ha sido aplastado y la pregunta silenciada no hay sujeto que se sostenga.

¿Es posible situar en el interior de la relación médico-paciente éste síntoma de la época en que vivimos? Del lado del médico encontramos la exigencia de la eficacia, de efectividad, en una época seducida por los resultados tecnocientíficos. El médico es sometido todo el tiempo a evaluaciones de eficacia. La eficacia se asocia a la rapidez del diagnóstico y al uso adecuado de la tecnología. La salud se ha convertido en una industria donde los procedimientos deben ser rentables. Los avances técnicos ya no pueden dejarse afuera por lo que, si no existe demanda de ellos, habrá que generarla. Pero, entonces, el paciente ya no es un sujeto, es un cuerpo que se presta a la confirmación de la efectividad de los avances de la biología. Y el médico queda atrapado en una operación, en el cumplimiento de una norma que hace callar la pregunta por el sentido.

Las normas son necesarias, como también lo es poder reconocer la locura que a veces engendra su aplicación. Aun cuando se trate de normas válidas, no podemos definir al sujeto por 
las reglas a las cuales obedece. Éstas sirven para orientarnos, dan una guía para la acción, pero nada dicen de la particularidad de los sujetos que obedecen a ellas. Ahora bien, en el cumplimiento de la norma es posible distinguir cómo el reglamento se adapta al caso y, fundamentalmente, captar lo que en el caso excede el reglamento. A partir de allí es posible dirigir la acción sin alienarla a una operación.

Por último, tampoco podemos reducir el reconocimiento de la singularidad del paciente al respeto por sus derechos lo cual es condición necesaria, pero no suficiente. Tomemos por ejemplo el principio de autonomía: "Se dice que una persona actúa con autonomía cuando tiene independencia respecto de controles externos y capacidad para obrar de acuerdo a una elección propia. La autonomía se prueba en las opciones escogidas y, por ello, la potencialidad de tenerla, si bien importante, se limita o restringe en numerosos casos [...] Normalmente, lo que se juzga al considerar la autonomía es el grado de intencionalidad de los actos, la comprensión que de ellos tiene el agente y la ausencia de coerciones o limitaciones(12)".

Aún cuando se afirme que se ha respetado la autonomía del paciente, nada se sabe de él. La alteridad del semejante, como vimos antes, no nos es accesible por la vía del saber, pues esto implicaría reducir al otro a la categoría de objeto del conocimiento. "La particularidad no sólo se alcanza respetando los derechos de la persona, lo que es un requisito necesario, sino dejando hablar al sujeto. Primero no hay que hablarlo, o someterlo a la regla, aunque fuera la mejor de las reglas (7, p.86)".

Aunque la regla fuera respetar la autonomía del paciente, es necesario que el médico pueda situarse frente al sujeto como frente a un misterio, para poder reconocer la particularidad de éste y para que el paciente pueda reconocerse en su particularidad, pues es también responsabilidad del paciente no someterse a la demanda de ofrecer su cuerpo como deshabitado de todo deseo.

Podría objetarse que cuando se dispone de sólo veinte minutos, o a veces menos, para hacer un diagnóstico y recomendar un tratamiento, poco espacio queda para que el paciente "hable". Pero esto sería reducir la palabra al mensaje. Se habla también con el cuerpo, en el síntoma que no ha pasado al registro de la palabra. Y el médico, que asume el desafío de reconocer su ignorancia con respecto a la alteridad del otro que es su paciente, puede "escuchar", atender al enfermo y no a la enfermedad. El paciente, así atendido, es un sujeto que puede sorprender y sorprenderse en una pregunta que, hasta el momento del encuentro con el médico, había quedado suspendida.

\section{Referencias}

1. Levinas E. El Tiempo y el Otro. Barcelona: Ediciones Paidós Ibérica; 1993: 109- 110.

2. Lacan J. Escritos I. Buenos Aires: Siglo XXI Editores; 2002: 94.

3. Castoriadis C. El taparrabos de la ética. Centro de Investigaciones en Bioética y Salud Pública (Universidad de Santiago de Chile) 1996; 1(2):1.

4. Engelhardt HT. Los fundamentos de la bioética. Barcelona: Ediciones Paidós; 1995: 16.

5. Mainetti JA. Jules Romains: Knock o la medicalización de la vida. Quirón 2001;1(32): 124. 
El malestar en la relación médico-paciente - G. Manitta

6. Ladrière J. Del sentido de la bioética. Acta Bioethica 2000; VI(2): 203.

7. Laurent E. Psicoanálisis y salud mental. Buenos Aires: Editorial Tres Haches; 2000: 144.

8. Tort M. El deseo frío. Buenos Aires: Ediciones Nueva Visión SAIC; 1994: 161.

9. Lacan J. Intervenciones y Textos 1. Buenos Aires: Ediciones Manantial SRL; 2002: 92.

10. Ladrière J. El reto de la racionalidad. Salamanca: Ediciones Sígueme; 1977: 63.

11. Hardt M, Negri A. Imperio. Buenos Aires: Paidós; 2003: 36.

12. Lolas F. Bioética. El diálogo moral en las ciencias de la vida. Santiago de Chile: Editorial Mediterráneo; 2001: 64 . 